ASME JOURNAL OF THERMAL SCIENCE AND ENGINEERING APPLICATIONS

Accepted March $29^{\text {th }} 2017$

IMPACT FACTOR 0.809

PUBLISHER: ASME, NEW YORK, USA.

ISSN: 1948-5085

\title{
Electrothermal Transport in Biological Systems: An Analytical Approach for Electrokinetically-Modulated Peristaltic Flow
}

\author{
${ }^{\# 1}$ Dharmendra Tripathi, ${ }^{1}$ Ashish Sharma ${ }^{2}$ O. Anwar Bég and ${ }^{1}$ Abhishek Tiwari \\ ${ }^{1}$ Department of Mechanical Engineering, Manipal University Jaipur, Rajasthan-303007, India. \\ ${ }^{2}$ Fluid Dynamics, Bio-Propulsion and Nanosystems, Department of Mechanical and Aeronautical \\ Engineering, Salford University, Newton Building, The Crescent, Salford, M54WT, England, UK.
}

\# Corresponding author: Email: dharmtri@gmail.com

\begin{abstract}
A mathematical model is developed to investigate the combined viscous electro-osmotic flow and heat transfer in a finite length micro-channel with peristaltic wavy walls. The influence of Joule heating is included. The unsteady two-dimensional conservation equations for mass, momentum and energy conservation with viscous dissipation, heat absorption and electro-kinetic body force, are formulated in a Cartesian co-ordinate system. The Joule heating term appears as a quadratic function of axial electrical field in the energy conservation equation. The axial momentum and energy equations are coupled via the thermal buoyancy term. The peristaltic waves propagating along the microchannel walls are simulated via a time-dependent co-sinusoidal wave function for the transverse vibration of the walls. Both single and train wave propagations are considered. Constant thermo-physical properties are prescribed and a Newtonian viscous model is employed for the fluid. The electrical field terms are rendered into electrical potential terms via the Poisson-Boltzmann equation, Debye length approximation and ionic Nernst Planck equation. The dimensionless emerging linearized electro-thermal boundary value problem is solved using integral methods. A parametric study is conducted to evaluate the impact of isothermal Joule heating term on axial velocity, temperature distribution, pressure difference, volumetric flow rate, skin friction and Nusselt number. The modification in streamline distributions with Joule heating and electro-osmotic velocity is also addressed to elucidate trapping bolus dynamics.
\end{abstract}

Keywords: Electro-kinetic pumping; peristalsis; heat transfer; Nusselt number; Debye length; Grashof number; heat generation/absorption; unsteady flow; trapping; micro-channel. 


\section{INTRODUCTION}

The continuous scaling down of engineering devices has resulted in significant advances in micro- and nano-fluidics. Electro-osmotic systems are increasingly being deployed in many areas of medicine and industrial technology [1]. Electro-osmotic flows are generated by the Coulomb electrical force produced by the application of an external electrical field. This mobilizes electrical charges in solution (electrolytes) in particular near highly charged surfaces. This phenomenon can be exploited in many complex systems including micro-pumping for integrated electronic circuits $[2,3]$, miniature direct methanol fuel cell (DMFC) energy systems [4], ocean thermal-osmotic energy conversion systems [5] (which include reverse electro-dialysis in which concentrated ionic solutions move through alternating cells and fresh water flows through other cells resulting in a voltage generated across each ion-exchange membrane) [6] and also petrochemical purification processes [7]. Electro-osmotic flows are also fundamental to numerous physiological processes including gel electrophoresis in tissue engineering [8], cerebral trans-diffusion phenomena [9] and tri-phasic tissue behaviour in synovial mechanics [10].

An important biophysical mechanism which is fundamental to many critical transport processes is peristalsis. This involves a rhythmic process of wave-like contractions of physiological vessel which propels contents efficiently over considerable distances. It arises in intestinal hydrodynamics [11], swallowing [12], ovum movement in the oviduct or uterus [13], endocardial cushioning in embryonic heart transition from peristaltic to pulsatile flow [14]. Many analytical and computational studies have been reported on peristaltic propulsion. Bertuzzi et al. [15] studied numerically the transport of a solid spherical bolus enclosed in a contractile membrane. Tripathi et al. [16] derived analytical solutions for the influence of curvature and non-Newtonian characteristics on transport of gastric fluids in peristaltic pumping. Tözeren et al. [17] investigated theoretically the viscous-dominated peristaltic transport of rigid particles along distensible conduits using a thin elastic shell model and lubrication theory.

In recent years peristaltic electro-osmotic (EO) pumps have been explored as a bioinspired design for improving electrokinetic systems. These designs minimize working parts and increase system efficiency and sustainability. Important works in this regard 
include the study by Hayakawa et al. [18] on gel electrophoresis preparation with EO micro-pumps in elution chamber design, Zhu et al. [19] for gas diffusion micro-chip enhancement and Yeh et al. [20] for dual electrode travelling-wave EO pump configurations. Mathematical studies of electro-osmotic peristaltic flows have also stimulated some interest, and serve as a compliment to experimental investigations. Chakraborty [21] considered the influence of axial electric field on microfluidic transport in peristaltic microtubes, showing that careful prescription of peristalsis wave characteristics and axial electrokinetic body force may successfully boost the timeaveraged flow rate in such systems. El-dabe et al. [22] recently studied the influence of a vertical alternating current and electric field on peristaltic pumping of a dielectric viscoelastic Oldroyd fluid in a mildly stenosed conduit. They evaluated the effects of many parameters including electrical Rayleigh number, Reynolds number, wave number and Weissenberg number on flow characteristics. Roth [23] explored a different application of electro-kinetic peristalsis, namely aerodynamics in discharge plasma systems, showing that peristaltic induction of neutral gas by a traveling electrostatic wave is an effective mechanism for flow acceleration. Tripathi et al. [24] derived analytical solutions for unsteady electro-osmotic peristaltic transport in a microchannel subjected to transverse magnetic field and axial electrical field, using lubrication and Debye approximations. They showed that stronger magnetic field discourages bolus growth and decelerates axial flow and flow rate but elevates pressure difference at low time values. They also found that lower Debye electrical length increases time-averaged flow rate but decelerates axial flow. Si and Jian [25] presented perturbation solutions for electromagneto-hydrodynamic (EMHD) viscoelastic flow in a two slit corrugated microchannel, simulating peristaltic waves as periodic sinusoidal waves with small amplitude and computing the influence of dimensionless wave number and relaxation time and retardation time on pumping characteristics. Recently Tripathi et al. [26] investigated the transient electro-osmotic Newtonian peristaltic pumping in cylindrical vessels of finite length, under Debye-Hückel linearization, noting that greater axial electrical field (i.e. larger Helmholtz-Smoluchowski velocity) suppresses pressure for train wave propagation whereas it elevates pressure for single wave propagation. They further observed that with increasing electro-osmotic parameter (decreasing Debye length), the 
volumetric flow rate rises and approaches the value for very thin EDL (as electro-osmotic parameter tends to infinity).

The above studies generally neglected thermal transport i.e. heat diffusion. It has been identified however that the inclusion of ionic thermo-diffusion can exert a significant influence on ionic distribution, local electrical potential and even free charge density and therefore modifies substantially electro-osmotic flows [27]. The heat-conducting nature of real blood flows and also the presence of thermal effects in real micro-channel systems in industrial devices make the inclusion of heat transfer effects important since thermodiffusion-induced free charge density may influence thermo-diffusion-induced electroosmotic velocity. Applications of electro-osmotic heat transfer include microbial fuel cells for carbon capture [28], polymer electrolyte membrane (PEM) fuel cells [29] and thermal band broadening in electro-chromatography [30]. The hydrodynamics of electroosmotically generated flow differs markedly from conventional pressure-driven flows and therefore inevitably heat transfer in EO transport will also be affected. A number of researchers have therefore addressed thermal electro-osmotic micro-fluidic systems. Zhou et al. [31] considered thermal effects on EO flow under an imposed temperature difference, incorporating temperature-dependent thermophysical and electrofluid properties as well as ion thermo-diffusion. They showed that temperature difference/gradient induces a modification in ionic concentration, electrical potential, and electro-osmotic velocity profiles from their isothermal counterparts, and accelerates electro-osmotic transport. Soong and Wang [32] analyzed thermal electro-kinetic flow in a microchannel under asymmetric boundary conditions with wall-sliding motion, asymmetric zeta potentials and different heat fluxes imposed at the channel walls. Mukhopadhyay et al. [33] simulated thermally and hydrodynamically fully developed combined pressure-driven and electroosmotic flow in a micro-channel with isoflux wall boundary conditions, showing that thermofluid characteristics are strongly altered by the asymmetric wall boundary conditions. Another important characteristic of thermal electrokinetic flows is Joule heating which occurs commonly due to the presence of electrical potential gradient and electrical current. It has an amplified influence in systems with high electrical potential gradients or with high ionic concentration buffer solutions, which makes it relevant to both physiological and engineering applications. 
Chen [34] examined convective heat transfer in pressure-driven electro-osmotic nonNewtonian flow in micro-tubes, observing that larger length scale ratio decreases Nusselt number at low pressure gradients and furthermore that singularities arise in Nusselt number distributions for surface cooling as the ratio of Joule heating to wall heat flux is adequately high with negative sign. Experimental studies of Joule heating effects in electro-osmotic thermo-fluid mechanics include Cetin and $\mathrm{Li}$ [35] for electrophoresis fused-silica capillary reactors, by Rathore [36] for open/packed electro-driven separation techniques of capillary electrophoresis (CE) and capillary electro-chromatography (CEC) and by Petersen [37] for microchip based electrophoretic capillary systems. Vocale et al. [38] used a finite element method to investigate the electro-osmotic heat transfer inside silicon elliptical cross-section micro-channels with Joule heating and H1 boundary conditions. They showed that with decreasing electro-kinetic diameter, Joule heating induces a more prominent change in temperature distribution whereas increasing electrokinetic diameter elevates the convective heat transfer. Escandón et al. [39] reported asymptotic and computational solutions for steady-state conjugate heat transfer in fully developed electro-osmotic non-Newtonian laminar flow with Joule heating, using the viscoelastic simplified Phan-Thien-Tanner (SPTT) constitutive equation.

In the present investigation, we consider unsteady peristaltic electro-kinetic flow and heat transfer in a finite length micro-channel in the presence of Joule heating, heat generation/absorption, thermal buoyancy and viscous dissipation effects. Both single and train wave propagation are considered. Using lubrication and electrical Debye linearization approximations, the transformed, dimensionless boundary value problem is solved analytically. The influence of isothermal Joule heating term on axial velocity, temperature distribution, pressure difference, volumetric flow rate, skin friction (wall shear stress function) and Nusselt number (wall heat transfer rate) are presented graphically for fixed values of Grashof (thermal buoyancy) number, HelmholtzSmoluchowski velocity, heat source/sink (absorption) parameter and other geometric parameters. Trapping phenomena are also addressed via streamline visualization. Detailed interpretation is provided. Applications of the current analysis include thermal electro-osmotic micro-pumps and also hemodynamic transport in medical engineering 
[40]. The study is relevant to enhanced thermal transport in micro-fluidic systems, chromatography and electrophoretic processes in physiology.

\section{MATHEMATICAL MODEL}

The geometric model for the electro-osmotically-modulated peristaltic transport through finite length $(L)$ microfluidics channel, as depicted in Fig.1, is mathematically simulated for dual wave scenarios, as:

$\bar{h}(\bar{x}, \bar{t})=a-\bar{\phi} \cos ^{2} \frac{\pi}{\lambda}(\bar{x}-c \bar{t}) \quad \forall \bar{x} \in[0, L]$, for train wave propagation,

$\bar{h}(\bar{x}, \bar{t})=\left\{\begin{array}{l}a-\bar{\phi} \cos ^{2} \frac{\pi}{\lambda}(\bar{x}-c \bar{t}), \text { if } t<x<t+1 \\ a-\bar{\phi}, \quad \text { if } x \in[0, t] \cup[t+1, L]\end{array}\right.$, for single wave propagation:

where $\bar{h}, \bar{x}, \bar{t}, a, \bar{\phi}, \lambda$ and $c$ represent transverse vibration of the wall, axial coordinate, time, half width of the channel, amplitude of the wave, wavelength and wave velocity respectively. The temperatures at the center line and the wall of the peristaltic channel are given as: $T=T_{0} \quad($ at $y=0), T=T_{1}($ at $y=h)$. In Fig. 1 the case of a single wave propagating from the left reservoir to the right reservoir with a wave velocity $c$ is illustrated. The pressures at the left and right reservoirs (inlet and exit, respectively) are denoted as $p_{0}$ and $p_{L}$ respectively.

The governing equations for unsteady, two-dimensional, viscous, incompressible electrokinetically-modulated peristaltic transport and heat transfer may be formulated as follows:

$$
\begin{aligned}
& \frac{\partial \bar{u}}{\partial \bar{x}}+\frac{\partial \bar{v}}{\partial \bar{y}}=0 \\
& \rho\left(\frac{\partial}{\partial \bar{t}}+\bar{u} \frac{\partial}{\partial \bar{x}}+\bar{v} \frac{\partial}{\partial \bar{y}}\right) \bar{u}=-\frac{\partial \bar{p}}{\partial \bar{x}}+\mu\left(\frac{\partial^{2} \bar{u}}{\partial \bar{x}^{2}}+\frac{\partial^{2} \bar{u}}{\partial \bar{y}^{2}}\right)+\rho g \alpha\left(T-T_{0}\right)+\bar{\rho}_{e} E_{x}, \\
& \rho\left(\frac{\partial}{\partial \bar{t}}+\bar{u} \frac{\partial}{\partial \bar{x}}+\bar{v} \frac{\partial}{\partial \bar{y}}\right) \bar{v}=-\frac{\partial \bar{p}}{\partial \bar{y}}+\mu\left(\frac{\partial^{2} \bar{v}}{\partial \bar{x}^{2}}+\frac{\partial^{2} \bar{v}}{\partial \bar{y}^{2}}\right) \\
& \rho c_{p}\left(\frac{\partial}{\partial \bar{t}}+\bar{u} \frac{\partial}{\partial \bar{x}}+\bar{v} \frac{\partial}{\partial \bar{y}}\right) T=k\left(\frac{\partial^{2} T}{\partial \bar{x}^{2}}+\frac{\partial^{2} T}{\partial \bar{y}^{2}}\right)+\mu \varphi+\Omega+\sigma E_{x}^{2},
\end{aligned}
$$




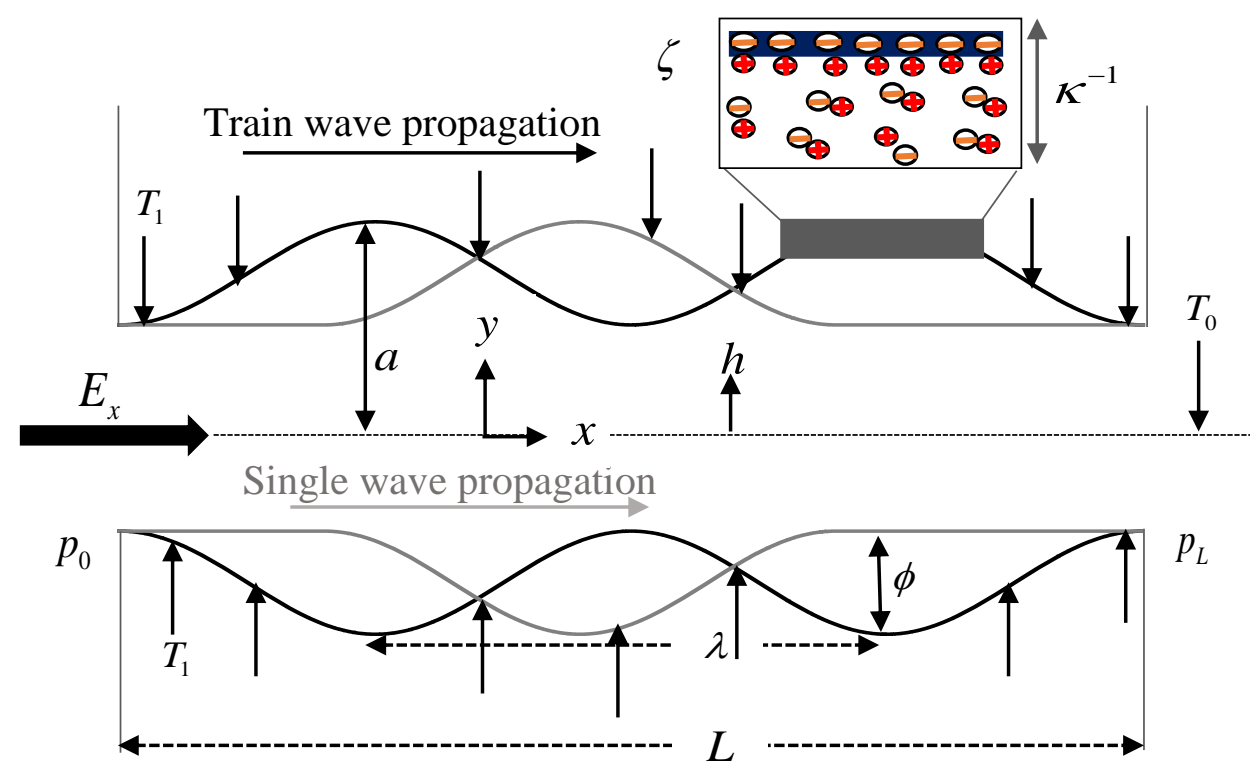

Figure 1. Schematic view of electro-osmotically-modulated peristaltic transport between two parallel plates subjected to constant temperature $\left(T=T_{1}\right)$ at the top and bottom surfaces.

Here $\rho, \bar{u}, \bar{v}, \bar{p}, \mu$, and $E_{x}$ denote the fluid density, axial velocity, transverse velocity, pressure, fluid viscosity, and electrokinetic body force and $g, \alpha, T, c_{p}, k, \varphi, \sigma$ and $\Omega$ denote the acceleration due to gravity, coefficient of linear thermal expansion of fluid, temperature, specific heat at constant pressure, thermal conductivity, viscous dissipation, the electrical conductivity of the buffer fluid and the heat absorption parameter. The first and second terms of Eq.(5) indicate the thermal energy transfer due to convection and thermal diffusion, respectively, while the third, fourth and fifth terms show thermal energy generation in the system due to viscous dissipation, heat absorption and Joule heating, respectively. The electric potential within the microchannel is given by according to well-known Poisson-Boltzmann equation as:

$\nabla^{2} \bar{\Phi}=-\frac{\bar{\rho}_{e}}{\varepsilon}$,

where $\varepsilon$ is the permittivity, $\bar{\rho}_{e}=e z\left(\bar{n}^{+}-\bar{n}^{-}\right)$is the electrical charge density, $\bar{n}_{+}$and $\bar{n}_{-}$ are positive and negative ions having bulk concentration (number density) $n_{0}$ and a valence of $z_{+}$and $z_{-}$respectively, and $e$ represents elementary charge. Further, in order 
to determine the potential distribution, it is necessary to describe the charge number density. For this, the ionic number distributions of the individual species are given by the Nernst-Planck equation for each species as:

$$
\frac{\partial \bar{n}_{ \pm}}{\partial \bar{t}}+\bar{u} \frac{\partial \bar{n}_{ \pm}}{\partial \bar{x}}+\bar{v} \frac{\partial \bar{n}_{ \pm}}{\partial \bar{y}}=D\left(\frac{\partial^{2} \bar{n}_{ \pm}}{\partial \bar{x}^{2}}+\frac{\partial^{2} \bar{n}_{ \pm}}{\partial \bar{y}^{2}}\right) \pm \frac{D z e}{k_{B} T}\left(\frac{\partial}{\partial \bar{x}}\left(\bar{n}_{ \pm} \frac{\partial \bar{\Phi}}{\partial \bar{x}}\right)+\frac{\partial}{\partial \bar{y}}\left(\bar{n}_{ \pm} \frac{\partial \bar{\Phi}}{\partial \bar{y}}\right)\right),
$$

Implicit in this analysis, is the assumption of equal ionic diffusion coefficients for both the species, and that the mobility of the species is given by the Einstein formula and $D$ represents the diffusivity of the ionic species, $k_{B}$ is Boltzmann constant. Introducing the following non-dimensional parameters; $\quad x=\frac{\bar{x}}{\lambda}, y=\frac{\bar{y}}{a}, t=\frac{c \bar{t}}{\lambda}, u=\frac{\bar{u}}{c}, v=\frac{\bar{v}}{c \delta}$, $h=\frac{\bar{h}}{a}, \quad p=\frac{\bar{p} a^{2}}{\mu c \lambda}, \quad \phi=\frac{\bar{\phi}}{a}, \Phi=\frac{\bar{\Phi}}{\zeta}, n=\frac{\bar{n}}{n_{0}}$, and the nonlinear terms in the Nernst Planck equations are $O\left(P e \delta^{2}\right)$, where $P e=R e S c$ represents the ionic Peclet number and $S c=\mu / \rho D$ denotes the Schmidt number. Therefore, the nonlinear terms may be dropped in the limit that $R e, P e, \delta<<1$ where $\operatorname{Re}=\frac{\rho c a \delta}{\mu}$ is Reynolds number and $\delta=\frac{a}{\lambda}$ wave number. In this limit, the Poisson equation is obtained as:

$$
\frac{\partial^{2} \Phi}{\partial y^{2}}=-\kappa^{2}\left(\frac{n_{+}-n_{-}}{2}\right)
$$

where $\kappa=a e z \sqrt{\frac{2 n_{0}}{\varepsilon K_{B} T}}=\frac{a}{\lambda_{d}}$, is known as the electro-osmotic parameter and $\lambda_{d} \propto \frac{1}{\kappa}$ is Debye length or characteristic thickness of electrical double layer (EDL).

The ionic distribution may be determined by means of the simplified Nernst Planck equations:

$$
0=\frac{\partial^{2} n_{ \pm}}{\partial y^{2}} \pm \frac{\partial}{\partial y}\left(n_{ \pm} \frac{\partial \Phi}{\partial y}\right)
$$

subjected to $n_{ \pm}=1$ at $\Phi=0$ and $\partial n_{ \pm} / \partial y=0$ where $\partial \Phi / \partial y=0$ (bulk conditions). These yield the much celebrated Boltzmann distribution for the ions:

$$
n_{ \pm}=e^{\mp \Phi}
$$


Combining equation (8) and (10), we obtain the Poisson-Boltzmann paradigm for the potential determining the electrical potential distribution:

$\frac{\partial^{2} \Phi}{\partial y^{2}}=\kappa^{2} \sinh (\Phi)$

In order to make further analytical progress, we must simplify equation (11). Equation (11) may be linearized under the low-zeta potential approximation. This assumption is not ad hoc since for a wide range of $\mathrm{pH}$, the magnitude of zeta potential is less than 25 $\mathrm{mV}$. Therefore, equation (11) may be simplified to give:

$\frac{\partial^{2} \Phi}{\partial y^{2}}=\kappa^{2} \Phi$

which may be solved subjected to $\left.\frac{\partial \Phi}{\partial y}\right|_{y=0}=0$ and $\left.\Phi\right|_{y=h}=1$, the potential function is obtained as:

$$
\Phi=\frac{\cosh (\kappa y)}{\cosh (\kappa h)}
$$

For the valuation of relative order of volumetric heat generation due to electric resistance heating (Joule heating), and a local volumetric heating due to viscous dissipation, one may obtain a ratio of strength of Joule heating and viscous dissipation as: $R_{v} \sim \frac{\sigma a \mu}{\kappa \varepsilon^{2} \zeta^{2}}$. We may consider the viscous dissipation to be negligible in comparison to Joule heating effects for channel width greater than $10 \mu \mathrm{m}$. The nonlinear terms in the momentum equation are found to be $O\left(\operatorname{Re} \delta^{2}\right)$, Re being the Reynolds number and $\delta$ denotes the ratio of the transverse length scale to the axial length scale. Therefore, the nonlinear terms may be dropped in the limit that $\operatorname{Re}, \delta \ll<$, the governing equations in a dimensionless coordinate system $(x, y)$ are reduced to:

$\frac{\partial u}{\partial x}+\frac{\partial v}{\partial y}=0$

$\frac{\partial p}{\partial x}=\frac{\partial^{2} u}{\partial y^{2}}+G r \theta+\kappa^{2} U_{H S} \Phi$,

$\frac{\partial p}{\partial y}=0$, 
$\frac{\partial^{2} \theta}{\partial y^{2}}+\beta+S=0$

where, $G r=\frac{g \rho^{2} \alpha a^{3}\left(T_{1}-T_{0}\right)}{\mu^{2}}$ is the Grashof number, $\theta=\frac{T-T_{0}}{T_{1}-T_{0}}$ is the dimensionless temperature, $U_{H S}=-\frac{E_{x} \varepsilon \zeta}{\mu c}$ is the Helmholtz-Smoluchowski velocity or maximum electro-osmotic velocity, $\beta=\frac{a^{2} \mu \Omega}{k\left(T_{1}-T_{0}\right)}$ is the dimensionless heat source/sink (absorption) parameter, and $S=\sigma E_{x}^{2} a^{2} / k\left(T_{1}-T_{0}\right)$ for constant wall temperature, is the normalized generation term that represents the ratio of Joule heating to surface heat flux. The imposed boundary conditions are:

$\left.\theta\right|_{y=0}=0,\left.\theta\right|_{y=h}=1,\left.\frac{\partial u}{\partial y}\right|_{y=0}=0,\left.u\right|_{y=h}=0,\left.v\right|_{y=0}=0,\left.v\right|_{y=h}=\frac{\partial h}{\partial t},\left.p\right|_{x=0}=p_{0},\left.p\right|_{x=L}=p_{L}$.

The solution for the temperature field of Eq. (17), using the boundary conditions (18), is obtained as:

$\theta=\frac{y\left\{2+(S+\beta)\left(h^{2}-h y\right)\right\}}{2 h}$.

Integrating Eq.(15) with respect to $y$, and implementing the boundary condition (18), the axial velocity emerges as:

$u=\frac{1}{2}\left(y^{2}-h^{2}\right) \frac{\partial p}{\partial x}+\frac{G r}{6}\left\{\frac{\beta+S}{4}\left(y^{4}+h^{4}-2 y^{3} h\right)-\frac{1}{h}\left(y^{3}-h^{3}\right)\right\}-U_{H S}\left\{\frac{\cosh (\kappa y)}{\cosh (\kappa h)}-1\right\}$.

Integrating the continuity equation with respect to $y$, and using Eq. (20) and boundary condition (18). The transverse velocity is obtained as:

$$
\begin{aligned}
v= & \frac{y}{6}\left\{\frac{\partial^{2} p}{\partial x^{2}}\left(3 h^{2}-y^{2}\right)+6 h \frac{\partial p}{\partial x} \frac{\partial h}{\partial x}\right\}+\frac{G r}{24} \frac{\partial h}{\partial x}\left\{\frac{\beta+S}{2}\left(y^{4}-8 h^{3} y\right)-\left(\frac{y^{4}}{h^{2}}+8 h y\right)\right\} . \\
& -U_{H S} \sinh (\kappa y) \frac{\tanh (\kappa h)}{\cosh (\kappa h)} \frac{\partial h}{\partial x} .
\end{aligned}
$$


Integrating Eq. (21) with respect to $x$, the pressure gradient is given by:

$$
\frac{\partial p}{\partial x}=\frac{1}{h^{3}}\left[G_{0}(t)+3\left\{\int \frac{\partial h}{\partial t} d x+U_{H S}\left(h-\frac{\tanh (\kappa h)}{\kappa}\right)\right\}\right]+\frac{G r}{8}\left\{3+\frac{7(\beta+S) h^{2}}{10}\right\} .
$$

where $G_{0}(t)$ is arbitrary function of $t$ to be evaluated by using the finite length boundary conditions (18). The pressure difference can be computed along the axial length by:

$$
\Delta p=p(x, t)-p(0, t)=\int_{0}^{x} \frac{\partial p}{\partial s} d s
$$

Using boundary conditions $\left.p\right|_{x=0}=p_{0},\left.\quad p\right|_{x=L}=p_{L}$ in Eq. (23), and rearranging the terms, $G_{0}(t)$ is expressed as:

$$
G_{0}(t)=\frac{\left(p_{L}-p_{0}\right)-\int_{0}^{L}\left[3 h^{-3}\left\{\int \frac{\partial h}{\partial t} d x+U_{H S}\left(h-\frac{\tanh (\kappa h)}{\kappa}\right)\right\}+\frac{G r}{8}\left\{3+\frac{7(\beta+S) h^{2}}{10}\right\}\right] d x}{\int_{0}^{L} h^{-3} d x} .
$$

Volumetric flow rate is defined as $Q(x, t)=\int_{0}^{h} u d y$, and integrating, in view of Eq. (20), yields:

$Q(x, t)=\frac{h^{3}}{3}\left\{\frac{G r}{8}\left(3+\frac{7(\beta+S) h^{2}}{10}\right)-\frac{\partial p}{\partial x}\right\}+U_{H S}\left(h-\frac{\tanh (\kappa h)}{\kappa}\right)$.

The transformations between a wave frame $\left(x_{w}, y_{w}\right)$ moving with velocity $(c)$ and the fixed frame $(x, y)$ are given by :

$$
x=x_{w}-c t, \quad y=y_{w}, \quad u=u_{w}+c, \quad v=v_{w},
$$

where $\left(u_{w}, v_{w}\right)$ and $(u, v)$ are the velocity components in the wave and fixed frame respectively.

The volumetric flow rate in the wave frame is given by

$$
q_{w}=\int_{0}^{h} u_{w} d y_{w}=\int_{0}^{h}(u-1) d y_{w},
$$

which, on integration, yields:

$$
q_{w}=Q-h .
$$

Averaging volumetric flow rate along one time period, we get 


$$
\bar{Q}=\int_{0}^{1} Q d t=\int_{0}^{1}\left(q_{w}+h\right) d t,
$$

which, on integration, yields

$$
\bar{Q}=q_{w}+1-\phi / 2=Q+1-h-\phi / 2 .
$$

Using Eqs. (20 \& 21), the stream function in the wave frame (obeying the CauchyRiemann equations, $u_{w}=\frac{\partial \psi}{\partial y_{w}}$ and $v_{w}=-\frac{\partial \psi}{\partial x_{w}}$ ) takes the form:

$$
\begin{aligned}
\psi & =-\frac{1}{2}\left(\frac{\bar{Q}-1+\phi / 2+h}{h^{3}}\right)\left(y^{3}-3 y h^{2}\right)-y-U_{H S}\left(\frac{\sinh (\kappa y)}{\kappa \cosh (\kappa h)}-y\right) \\
& +\frac{G r}{48}\left\{\left(3+\frac{7(\beta+S) h^{2}}{10}\right)\left(y^{3}-3 h^{2} y\right)+(\beta+S)\left(\frac{2 y^{5}}{5}+2 h^{4} y-y^{4} h\right)-\frac{2}{h}\left(y^{4}-4 h^{3} y\right)\right\} .
\end{aligned}
$$

The shear stress, and heat transfer rate at the wall are defined in terms of skin friction coefficient $\left(C_{f}\right)$, and Nusselt number $(\mathrm{Nu})$ and obtained as:

$$
\begin{aligned}
& C_{f}=-\pi \phi h \sin (2 \pi(x-t))\left\{\left\{-\frac{\partial p}{\partial x}+\frac{G r}{12}\left(6+h^{2}(S+\beta)\right)\right\}+U_{H S} \kappa \tanh (\kappa h)\right\}, \\
& N u=-\frac{\pi\left(-2+h^{2}(S+\beta)\right) \phi \sin 2 \pi(x-t)}{2 h} .
\end{aligned}
$$

All the above results will reduce to the results reported in Ref.[42] for $U_{H S}=0 \& S=0$ and they are further reduced to the results of Ref. [43] for $U_{H S}=0, G r=0 \& S=0$

\section{NUMERICAL RESULTS AND DISCUSSION}

Numerical evaluations of the closed-form solutions derived in Secn. 2 are visualized in Figs. 2-10. These provide a perspective of the sensitivity of the regime to various electricphysical and thermo-physical parameters. Generally in these figures we consider a peristaltic wave amplitude of $\phi=0.6$ and heat generation $\beta=2$ which represent working conditions in real systems.

Fig. 2 illustrates the evolution of temperature with Joule heating parameter $(S)$. The temperature is greater for negative values of Joule heating parameter $(S)$ however it is lower for positive values of Joule heating parameter. The presence of an applied voltage gradient and its induced electric conduction current manifests in Joule heating in the 
fluid. For $S<0$ this results in volumetric energy generation in the electro-osmotic flow. However for $S>0$ energy is removed from the flow. This results in a cooling in the micro-channel electrokinetic flow with positive $S$ and a heating with negative $S$. the influence of Joule heating (whether assistive or opposing) to thermal distribution is clearly non-trivial. A similar observation has been made by Laser et al. [3] and also Chen [34], the latter noting a particularly strong reduction in temperature and enhancement in wall heat transfer rates (heat being conducted away from the fluid to the channel walls).

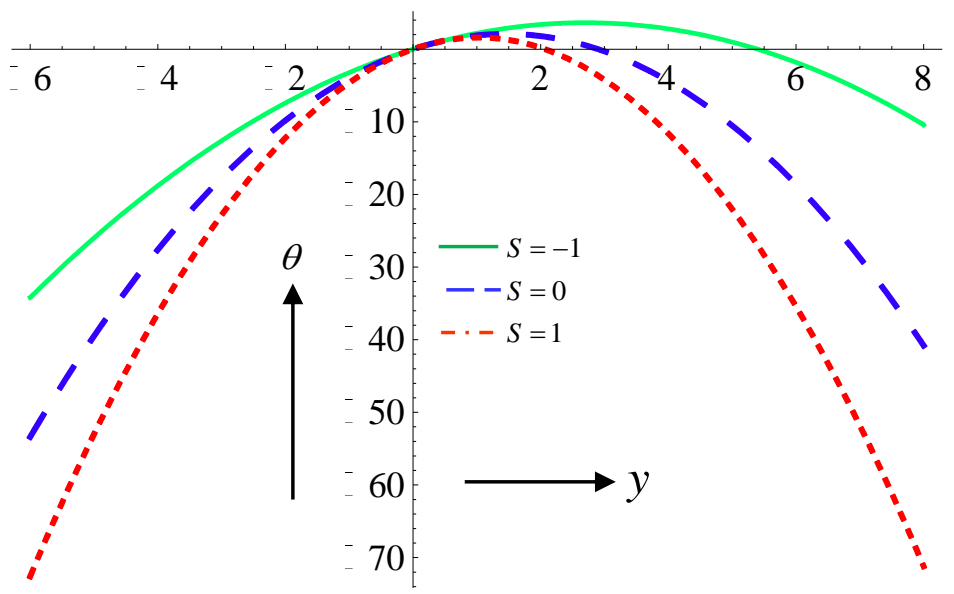

Fig.2. Temperature profile at $\phi=0.6, \beta=2, p_{x}=1, U_{H S}=1, G r=1, \kappa=1$ for different values of Joule heating parameter.

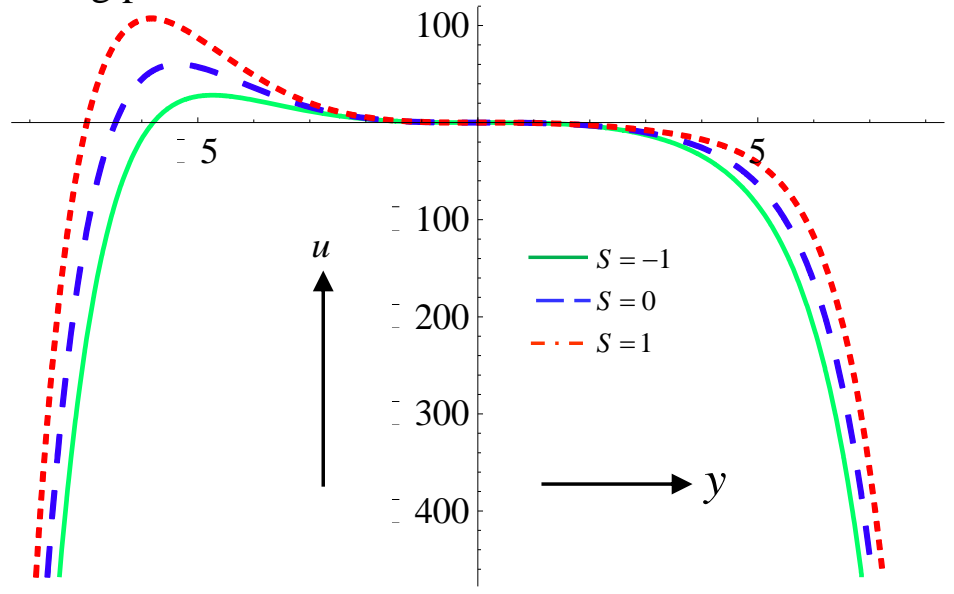




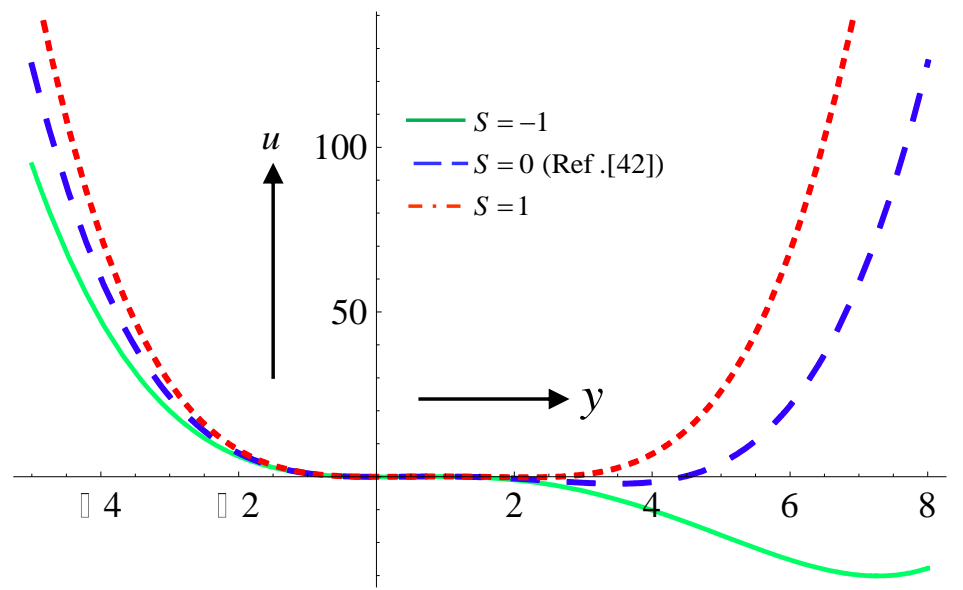

Fig.3. Velocity profile at $\phi=0.6, \beta=2, p_{x}=1, G r=1, \kappa=1$ for different values of Joule heating parameter (a) $U_{H S}=1$ (b) $U_{H S}=0$.
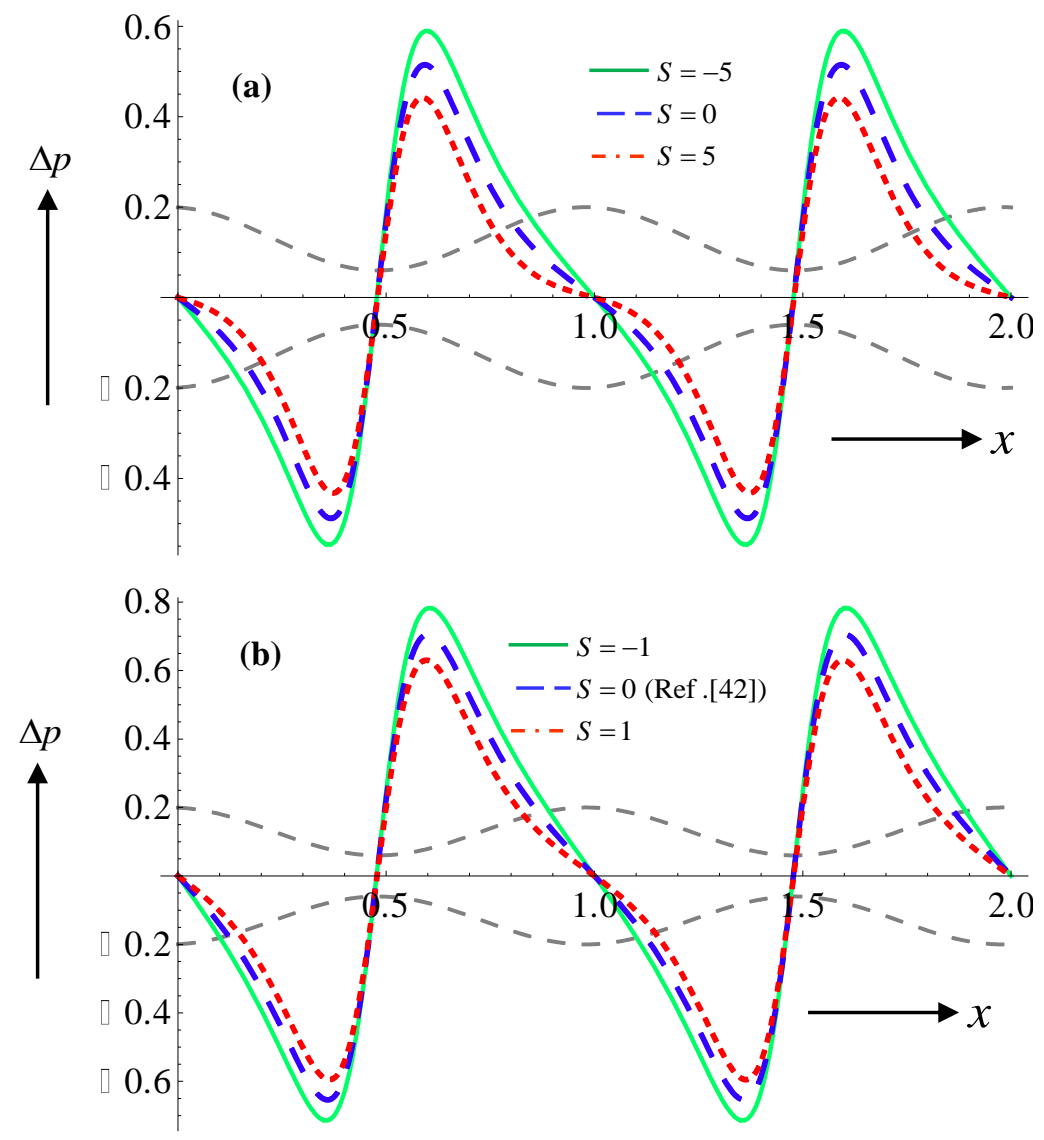


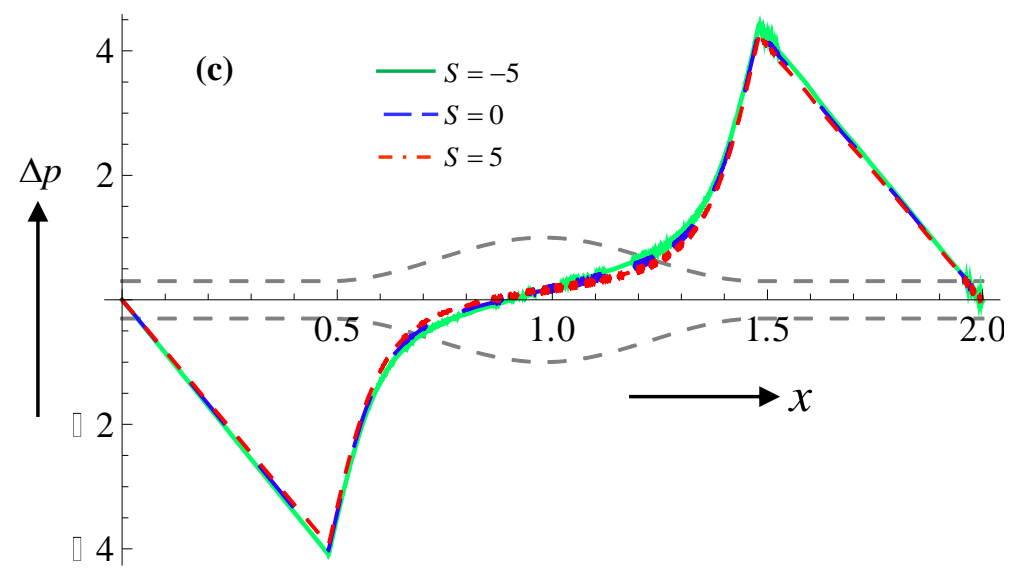

Fig.4. Pressure distribution along the channel length at $\phi=0.7, t=0.48, \beta=5$, $G r=1, \kappa=1$ for different values of Joule heating parameters (a) train wave propagation $U_{H S}=1$ (b) train wave propagation $U_{H S}=0$ (c) single wave propagation.
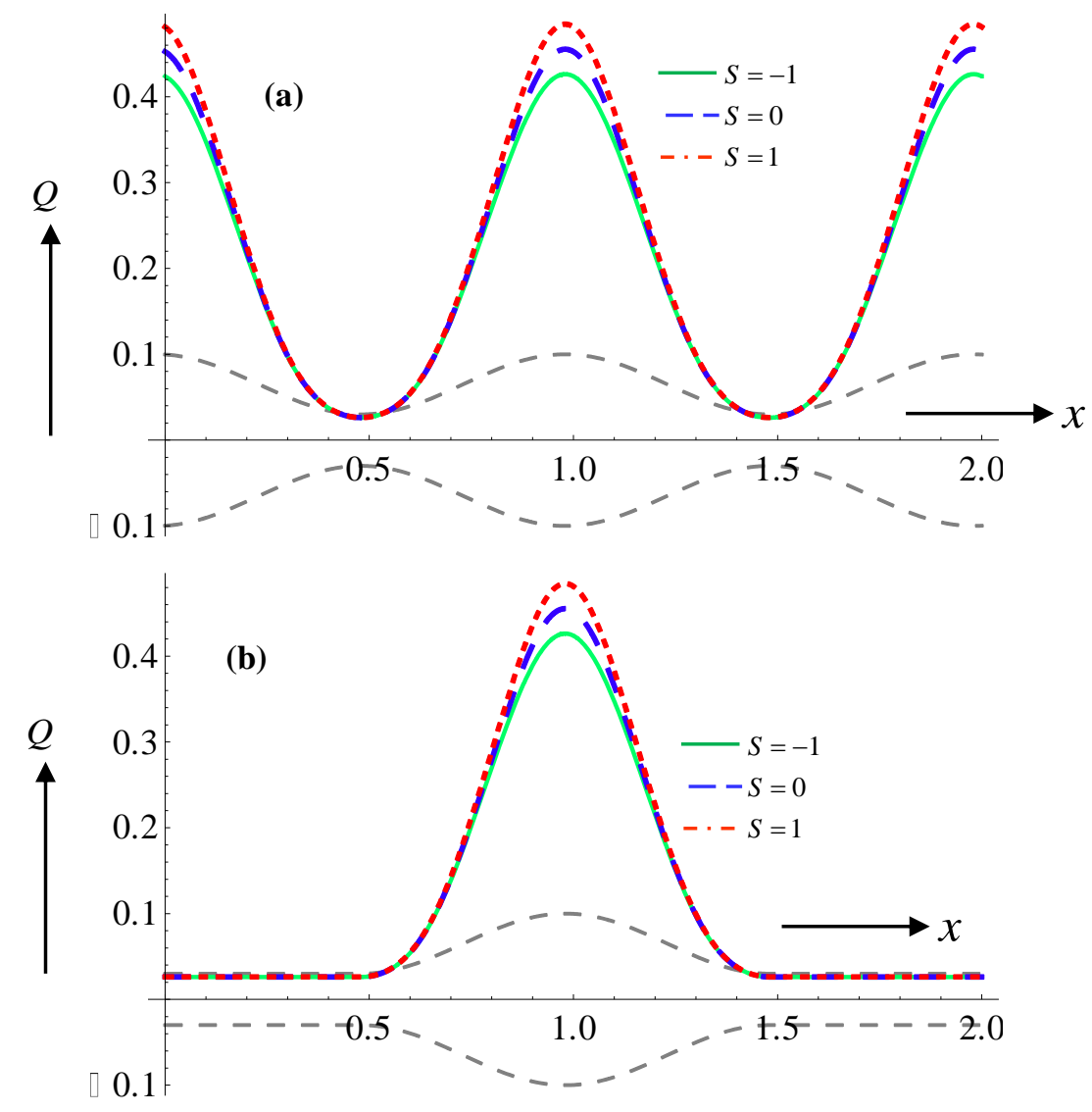

Fig.5. Volumetric flow rate along the channel length at $\phi=0.7, t=0.48, p_{x}=1, \beta=5, U_{H S}=1, G r=1, \kappa=2$ for different values of Joule heating parameters (a) train wave propagation (b) single wave propagation. 

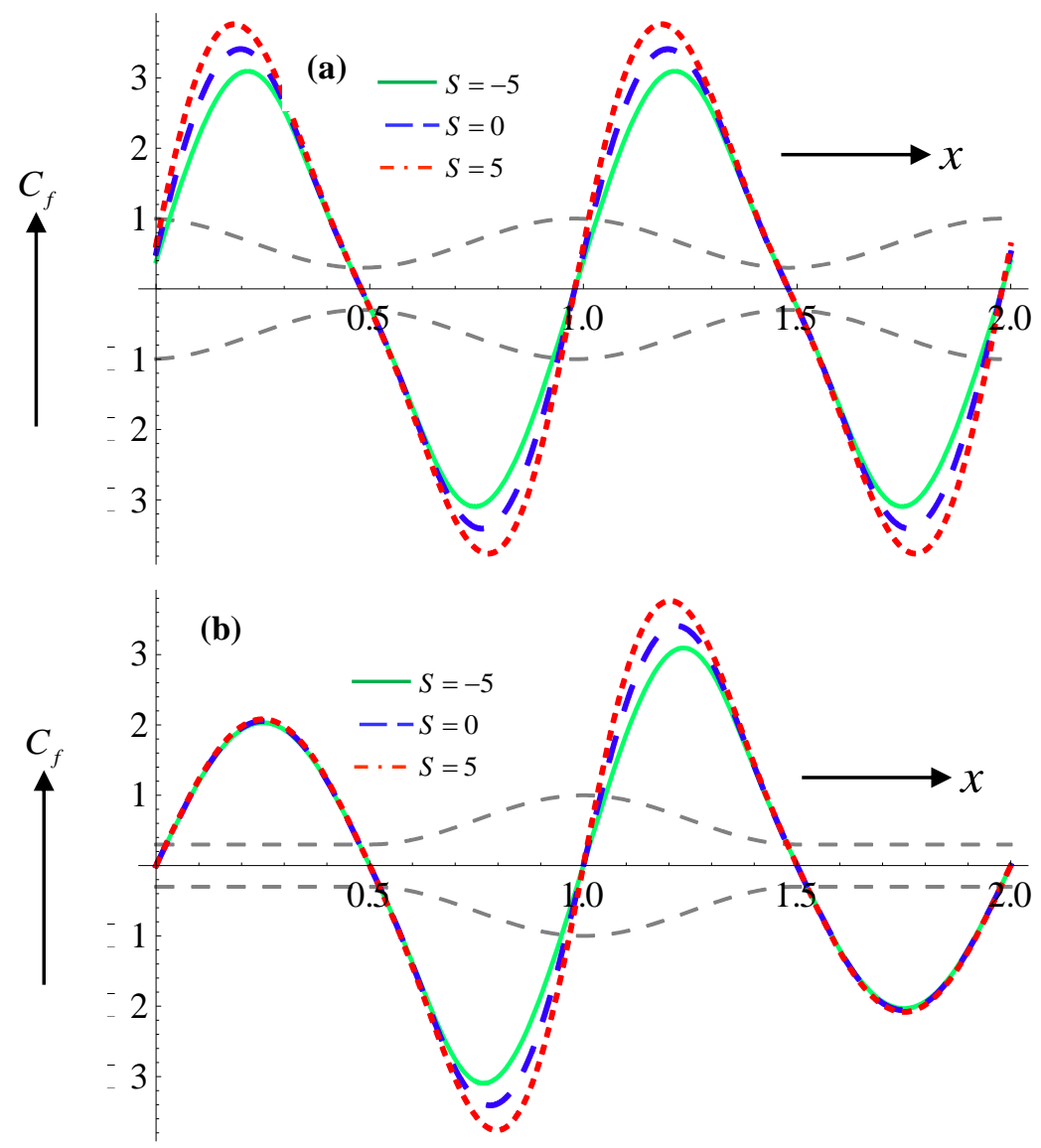

Fig.6. Skin friction coefficient along the channel length at $\phi=0.7, t=0.48, p_{x}=1, \beta=5$, $U_{H S}=1, G r=1, \kappa=2$ for different values of Joule heating parameters (a) train wave propagation (b) single wave propagation.

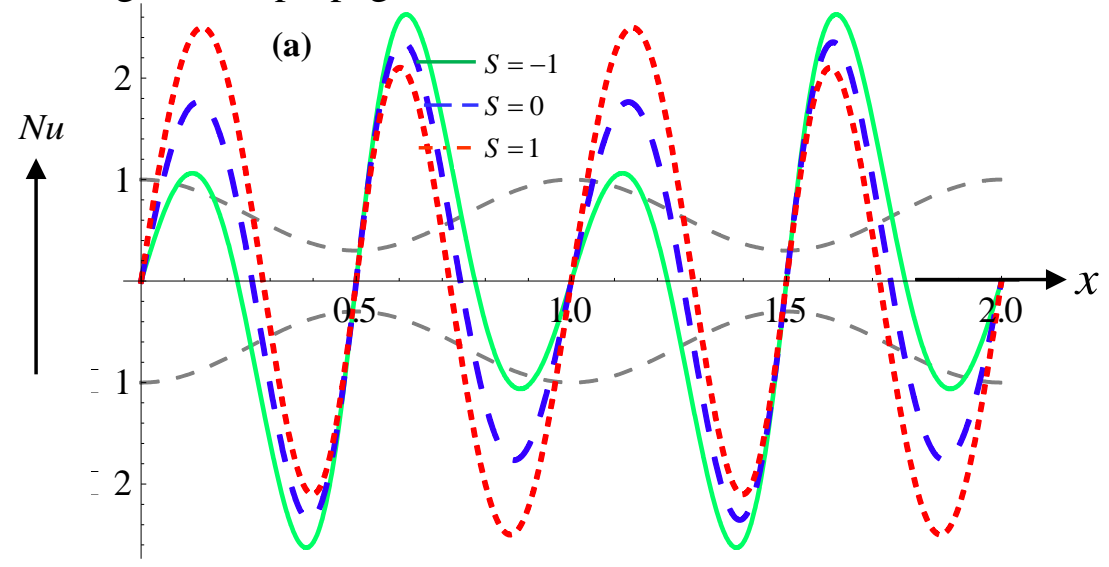




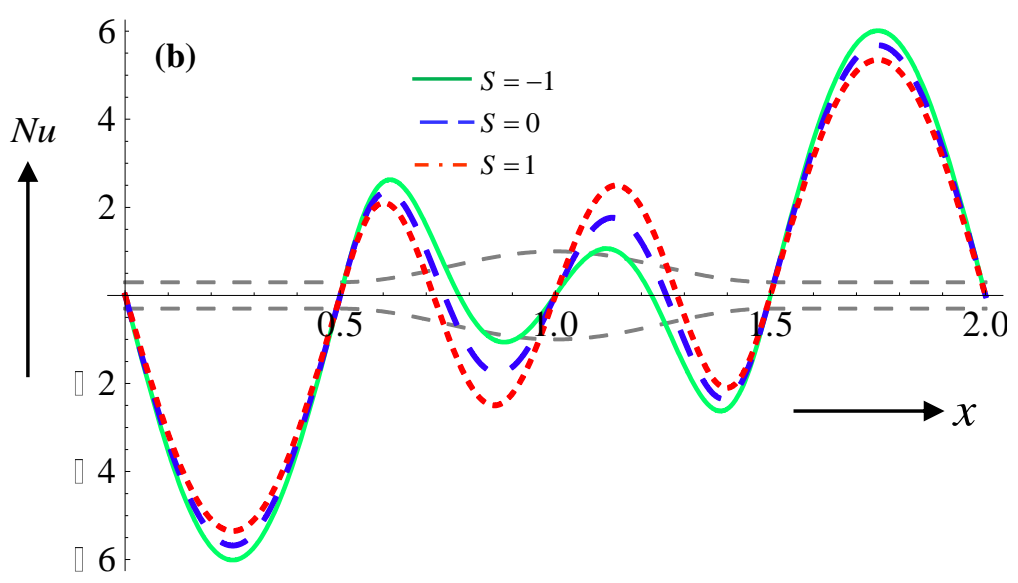

Fig.7. Nusselt number along the channel length at $\phi=0.7, t=0.48, \beta=5$ for different values of Joule heating parameters (a) train wave propagation (b) single wave propagation.

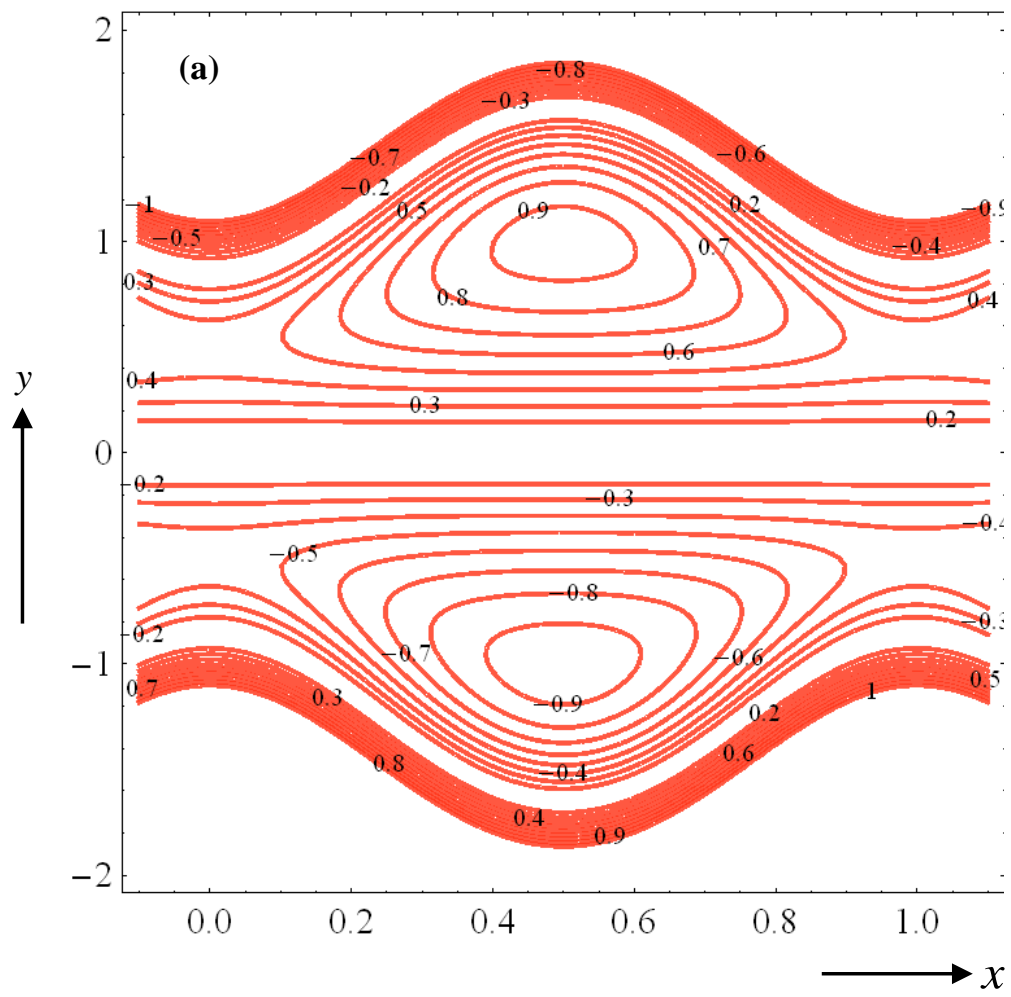



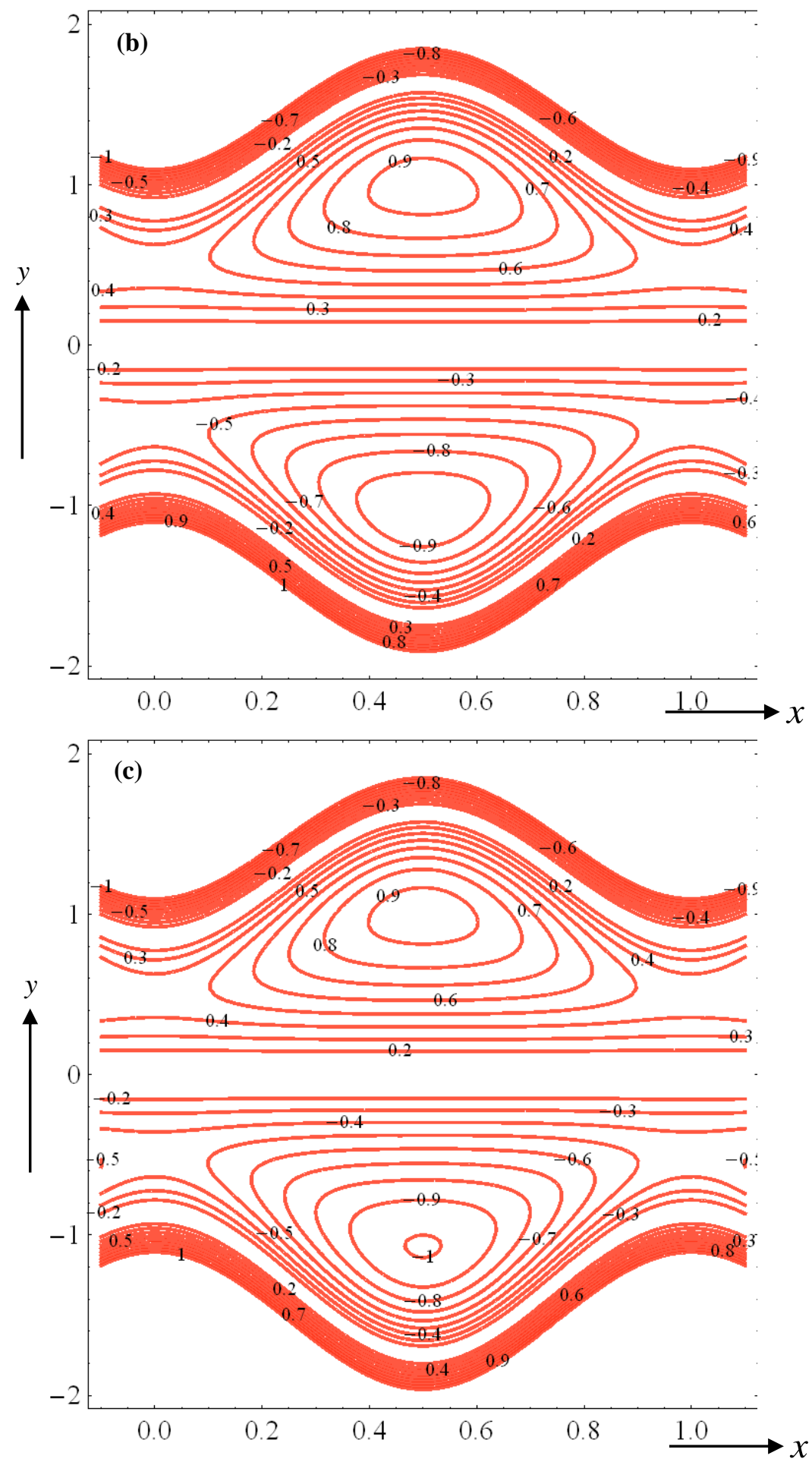

Fig.8. Stream lines at $U_{H S}=1, \phi=0.5, \bar{Q}=0.7, \beta=5, G r=0.1, \kappa=2$ for (a) $S=-5$, (b) $S=0$, (c) $S=5$. 

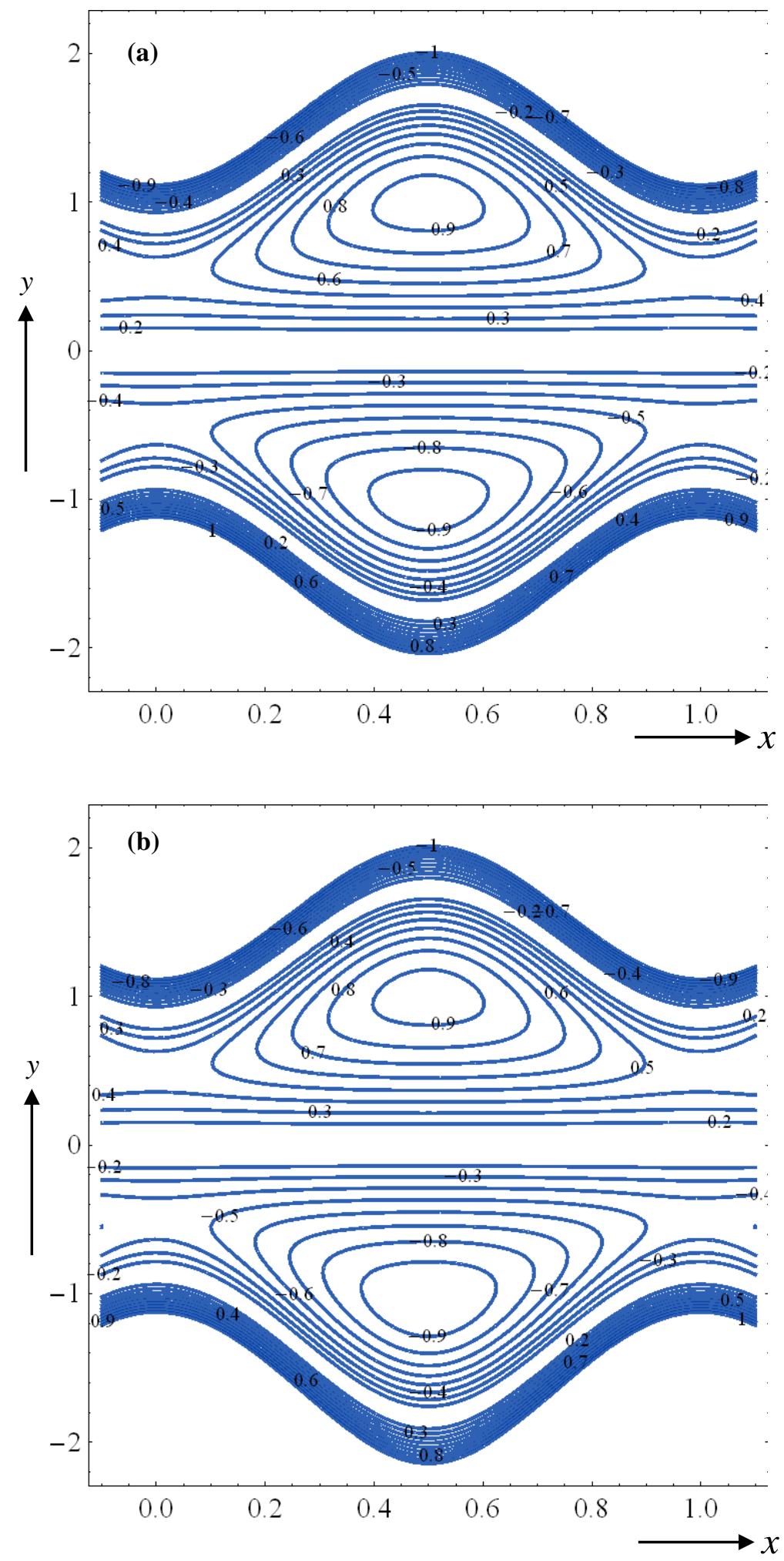


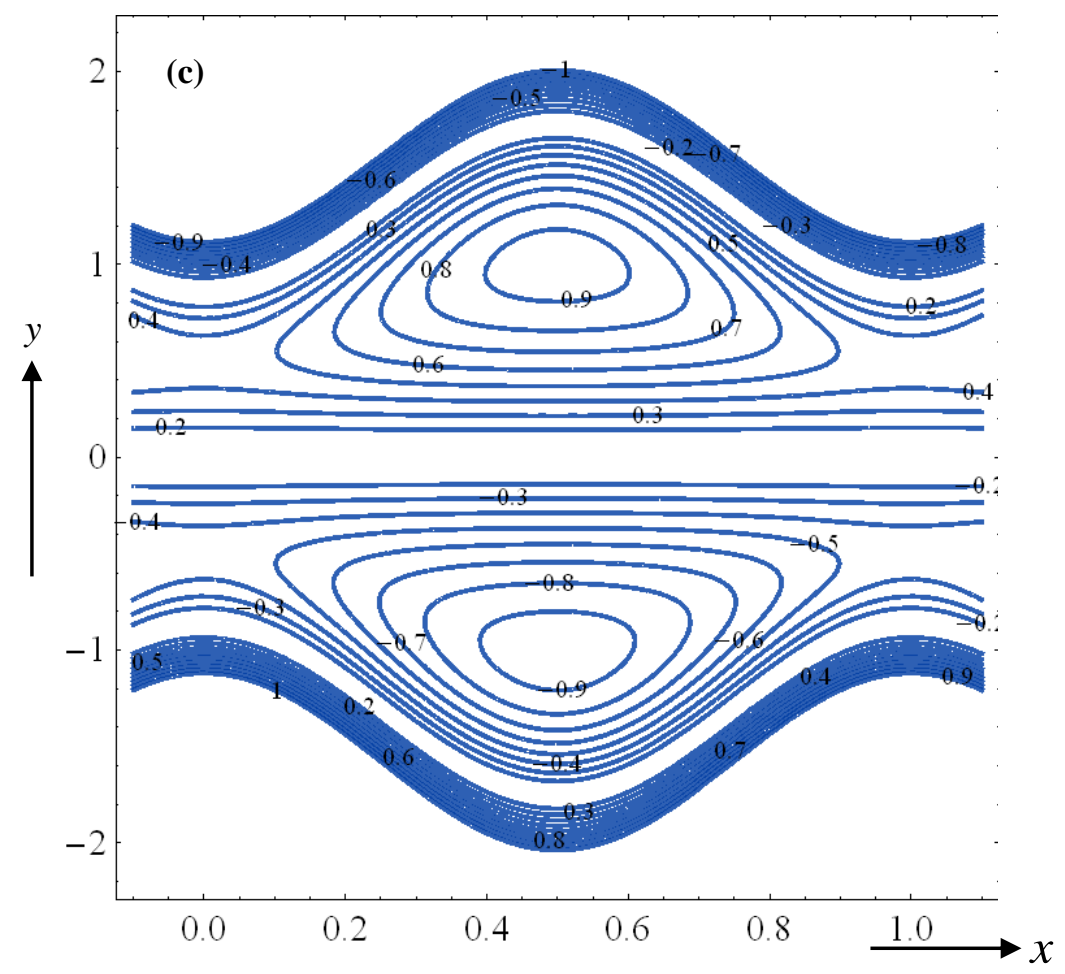

Fig.9. Stream lines at $U_{H S}=0, \phi=0.5, \bar{Q}=0.7, \beta=5, G r=0.1, \kappa=2$ for (a) $S=-5$, (b) $S=0$, (c) $S=5$.

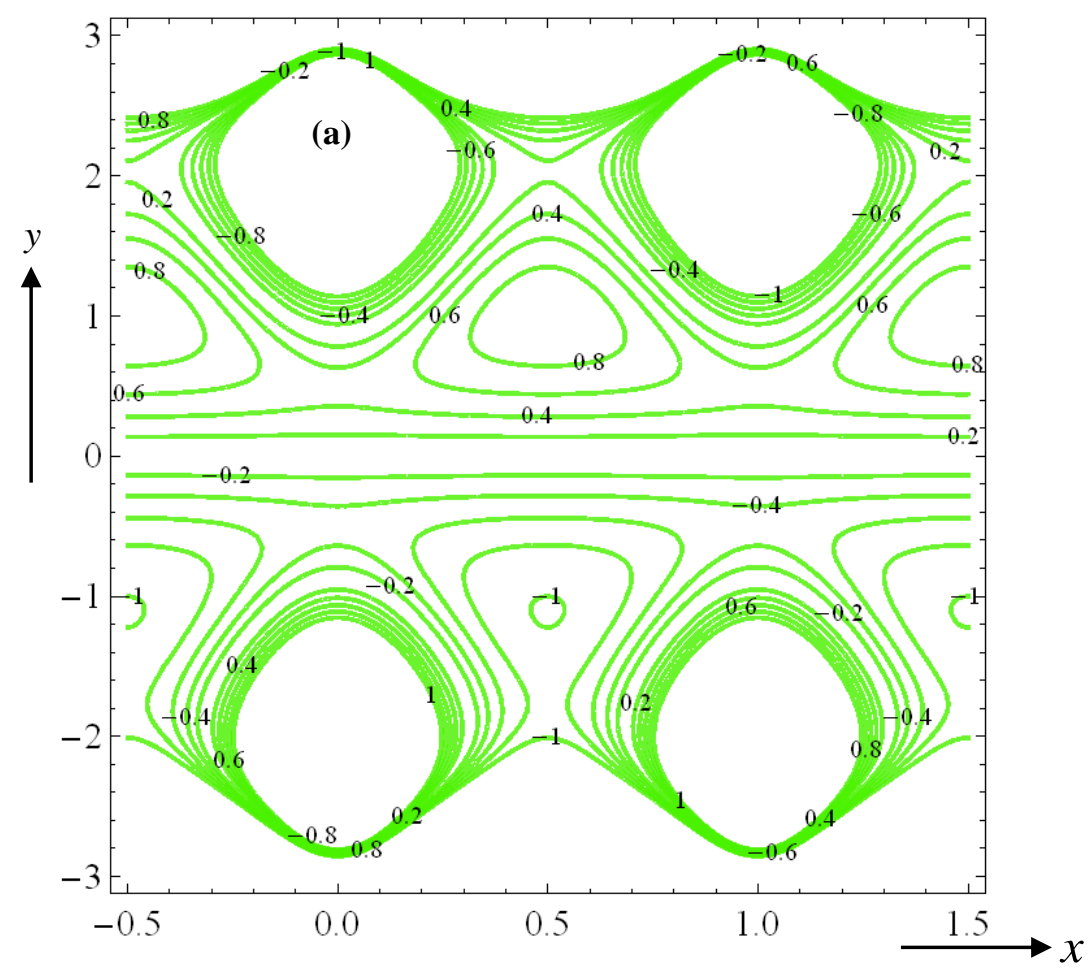



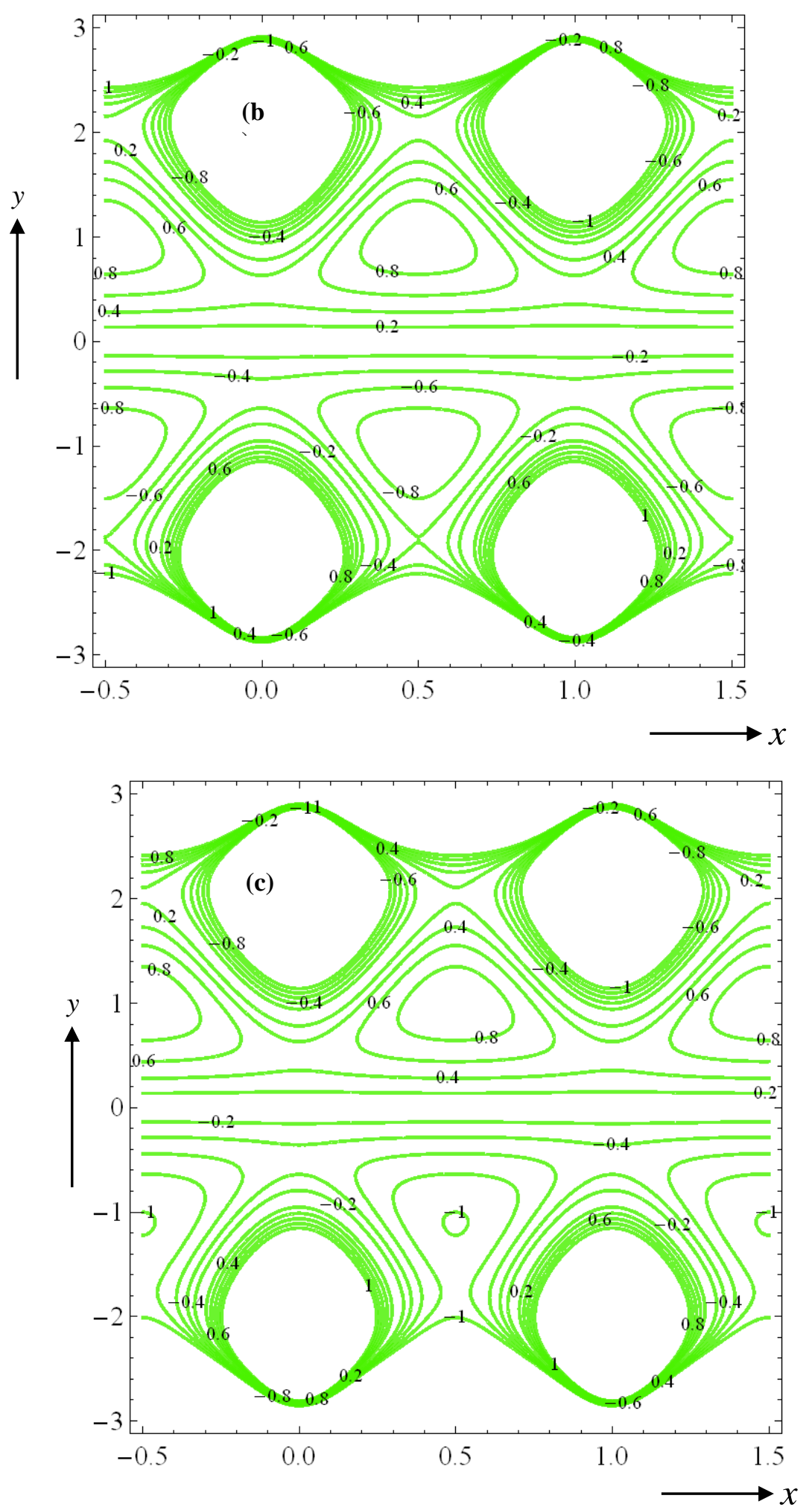

Fig.10. Stream lines at $U_{H S}=-1, \phi=0.5, \bar{Q}=0.7, \beta=5, G r=0.1, \kappa=2$ for (a) $S=-5$, (b) $S=0$, (c) $S=5$. 
Joule heating is associated with electric field-induced internal heating in the fluid under an externally applied potential gradient. Greater higher electrical field strength results in higher heat generation which heats the fluid, but apparently only for negative values of $S$. $S=\sigma E_{x}^{2} a^{2} / k\left(T_{1}-T_{0}\right)$ and is proportional to both the square of the applied axial electrical field and also the electrical conductivity of the electrolyte. This implies that greater molar concentration is also associated with greater heat generation. The overall influence of negative Joule heating is to heat the fluid and cool the micro-channel walls ("surface cooling") whereas for positive Joule heating the opposite effect is induced i.e. cooling of the fluid and heating of the micro-channel walls ("surface heating"). It is also noteworthy that generally we consider the following default values for other parameters i.e. $p_{x}=1$, $U_{H S}=1, G r=1, \kappa=1$ which correspond to unity axial pressure gradient in the positive xdirection, intermediate axial electrical field strength, equivalent thermal buoyancy and viscous forces, and unity value of electro-osmotic parameter.

Fig. 3a presents the axial velocity response to variation in Joule heating parameter $(S)$. A substantial acceleration in axial flow accompanies an increase in positive $S$ values with the contrary response for increasing negative $S$ values. Distributions are however found to be skewed and asymmetric about the channel centre line. The presence of the negative ion charges on the wall surfaces creates a concentration gradient of positively charged ions near the wall surfaces. This results in an electrical potential distribution in the electrolyte which we term the electric double layer (EDL). When electrical field (axial) is elevated, the Joule parameter is quadratically increased (for constant temperature difference) and the electro-kinetic body force (in the momentum eqn. (15) i.e. $+\kappa^{2} U_{H S} \Phi$ ) is also significantly enhanced. This results in an acceleration in the axial flow when $S>0$ (assistive case) and a deceleration in the flow when $S<0$ (retarding case). Flow control is therefore achieved with Joule heating. With greater acceleration in the flow, there will also be elevated dragging of ions with the fluid molecules along the micro-channel walls. Membrane, under constant pressure gradient $\left(p_{x}=1\right)$. Fig. $3 b$ present a comparative study between the present results $(S==1,-1)$ and results $(S=0)$ from Ref.[42]. 
Figs. 4a-c present the evolution in pressure difference $(\Delta p)$ with axial coordinate again for various Joule heating parameter values $(S)$. Both train wave and single peristaltic waves are considered. The simulation is visualized at $t=0.48$. A strong depression (fig. 4a) in pressure difference peaks accompanies a positive increase in $S$ values whereas peaks are markedly enhanced with negative $S$ values. The magnitudes of $S$ are also quite high. For $S=5$ the Joule heating is five times greater than the surface heat flux. A generally regular distribution of alternating peaks and troughs is computed for the train wave case. Fig.4b validates the results with Ref.[42]

However a significantly skewed pattern is computed for the single wave scenario (fig. 4c). Pressure difference is less dramatically influenced by Joule heating parameter for the single wave case as compared with the multiple (train) wave case. Peaks are also much sharper (triangular profiles are observed) for the single wave case whereas they are parabolic for the train wave case. The influence of positive Joule heating parameter on pressure difference is generally the opposite to that on velocity profiles i.e. it induces suppression of pressure differences whereas it results in enhancement of axial flow. The results concur strongly with other investigations including Soong and Wang [32].

Fig. 5a,b depict the profiles of volumetric flow rate $(Q)$ with axial coordinate (for both train wave and single peristaltic wave propulsion) with different values of Joule heating parameter values $(S)$. Both graphs are again plotted at $t=0.48$. The axial flow acceleration with greater electrical field and therefore larger positive $S$ values will boost flow rates. This results in an elevation in $Q$ values for $S=1$ and a reduction in $Q$ values for $S=-1$ (the case of $S=0$ corresponds to an absence of Joule heating). Whereas both peaks and troughs arise periodically for the train wave case (fig. 5a) only a single peak is observed for the single wave case (fig. 5b) i.e. the flow rate profile is plateau-like either side of the peak. However a distinct increase in flow rate is observed also for the single wave case. The number of peristaltic waves propagating along the micro-channel walls therefore imparts a significant modification to the volumetric flow rate, irrespective of the Joule parameter effect.

Figs. 6a,b present the skin friction (wall shear stress function) distributions with axial coordinate for various Joule heating parameter values $(S)$. As anticipated the skin friction 
magnitudes are enhanced with positive $S$ values (since the axial flow is accelerated) and reduced with negative $S$ values (axial flow retardation). However whereas in the train wave case (fig. 6a) peaks are consistently maintained at similar magnitudes along the channel length, in the single wave case (fig. 6b) the peak values strongly grow with distance along the channel $x$-axis. The influence of the sign of Joule heating is also significantly less prominent in the single wave case compared with the multiple (train) wave case, in particular at low $x$-values i.e. in the vicinity of the entry zone to the channel. Both cases however exhibit alternating profiles. The results appear to agree with the findings of other researchers, notably Chakraborty [21] although he did not consider coupled thermal effects.

Figs. 7a,b illustrate the Nusselt number profiles with axial coordinate for various Joule heating parameter values $(S)$. Nusselt number quantifies the heat transfer rate at the micro-channel walls. It defines also the ratio of thermal convective heat transfer to thermal conduction heat transfer. With enhanced positive Joule heating $(S>0)$, the electrolyte regime in the channel is cooled (temperatures are lower, as observed in fig. 2) and this results in enhanced diffusion of heat away from the fluid to the channel walls. Nusselt number is therefore elevated at lower values of $x$. The profiles for both train and single wave scenarios are found to alternate with progression along the channel length. The impact of Joule heating is therefore also different at different $\mathrm{x}$-values. Nusselt number profiles are consistently of similar shape in the train wave case (fig. $7 \mathrm{a}$ ) whereas they progressively increase in magnitude with axial distance for the single wave case (fig. 7b). Similar results have been reported by Sadeghi et al. [41].

Figs. 8-10 depict the collective influence of the Helmholtz-Smoluchowski velocity $\left(U_{H S}\right)$ and Joule heating parameter $(S)$ on trapping phenomena. In these plots, figs. 8a, 9a, 10a correspond to $S=-5$ with $U_{H S}=1,0$ and -1 , respectively. Figs $8 \mathrm{~b}, 9 \mathrm{~b}, 10 \mathrm{~b}$ correspond to $S=$ 0 with $U_{H S}=1,0$ and -1, respectively. Figs 8c, 9c, 10c correspond to $S=5$ with $U_{H S}=1$, 0 and -1 , respectively. With an increase in Helmholtz-Smoluchowski velocity $\left(U_{H S}\right)$ the axial electrical field is stronger. This weakly intensifies the circulation as $U_{H S}$ decreases from 1 to 0 . However with further decrease the dual bolus formation in figs $8 \mathrm{a}, 9 \mathrm{a}$ is further split into two pairs of boluses, with multiple vorticity zones appearing in fig. 10a. These trends are also observed in figs. Figs 8b,9b, 10b $(S=0)$ and also Figs. 8c, 9c, 10c 
$(S=5)$. The influence of maximum electro-osmosis velocity is therefore quite prominent. To elucidate the Joule heating effect, we consider figs. 8a-c, 9a-c and 10a-c. There is no tangible modification in the trapping bolus structures computed for figs 8a-c, figs. 9a-c or figs 10a-c with a dramatic increase in $S$.

\section{CONCLUSIONS}

Closed form analytical solutions have been derived for the influence of Joule electrothermal heating on peristaltic viscous electro-osmotic flow and heat transfer in a finite length micro-channel in the presence of viscous dissipation and heat absorption. Both single and train (multiple) wave propagation scenarios have been studied. The low Reynolds number and Debye approximation have been utilized. A finite length channel has been considered. Numerical evaluation of the solutions for axial velocity, temperature distribution, pressure difference, volumetric flow rate, skin friction (wall shear stress function) and Nusselt number (wall heat transfer rate) have been plotted using Mathematica software. The computations have shown that:

- With negative electro-osmotic velocity the dual bolus formation present for positive electro-osmotic velocity is modified to two pairs of boluses, with new circulation zones.

- A change in Joule heating parameter has no significant influence on the trapping phenomena.

- Increasing positive Joule parameter decreases temperatures (induces cooling) whereas increasing negative Joule number enhances temperatures (induces heating).

- Increasing positive Joule parameter accelerates the axial flow whereas increasing negative Joule parameter retard the axial flow in the micro-channel.

- Increasing positive Joule parameter reduces pressure differences for the train wave case, whereas the converse behavior is induced with negative $S$ values.

- Pressure differences are more evenly distributed for the train wave case compared with the single wave case and in the latter Joule heating has a much weaker influence. 
- Skin friction magnitudes are increased with positive Joule parameter and retarded with negative Joule parameter.

- Nusselt number is increased with positive Joule heating effect manifesting in heating of the micro-channel walls, with the opposite response for negative Joule heating effect.

The present work has been confined to Newtonian electrolytes which is applicable to study the heat transfer and electrokinetic transport in physiological systems. This model is also helpful in designing the microfluidics devices for biomedical engineering.

\section{ACKNOWLEDGEMENTS}

The authors are grateful to all the reviewers for their insightful comments which have served to improve the article.

\section{REFERENCES}

1. P.K. Dasgupta, L. Shaorong, Electroosmosis: a reliable fluid propulsion system for flow injection analysis, Anal. Chem. 66, 1792-1798 (1994).

2. Z. Shulin, Fabrication and characterization of electrokinetic micro-pumps, Thermomechanical Phenomena in Electronic Systems-Proc. Intersociety Conference, Las Vegas, Nevada, USA (2000).

3. J. Laser, A. M. Myers, S. Yao, K. F. Bell, K. E. Goodson, J. G. Santiago and T. W. Kenny, Silicon electroosmotic micropumps for integrated circuit thermal management, , $12^{\text {th }}$ Int. Conf. Solid State Sensors, Actuators and Microsystems, Boston, USA, June 8-12 (2003).

4. C. Buie, D. Kim, S. Litster and J. G. G. Santiago, Free convection direct methanol fuel cells powered by electroosmotic pumps, ElectroChemical Society (ECS) Trans. 3, 1279-1287 (2006).

5. H.H.G. Jellinek, H. Masuda, Osmo-power-theory and performance of an osmopower pilot plant, Ocean Engineering, 8, 103-128 (1981).

6. R.E. Lacey, Energy by reverse electrodialysis, Ocean Engineering, 7, 1-47 (1980). 
7. J. S. Eow, M. Ghadiri and A. O. Sharif, Electro-hydrodynamic separation of aqueous drops from flowing viscous oil, J. Petroleum Science and Engineering, 55, 146-155 (2007).

8. M. D. Hunckler, J. M.R. Tilley, R. K. Roeder, Molecular transport in collagenous tissues measured by gel electrophoresis, J. Biomechanics, 48, 4087-4092 (2015).

9. G. Li and B. M. Fu, An electrodiffusion model for the blood-brain barrier permeability to charged molecules, ASME J Biomech Eng., 133(2) 021002 (2011).

10. W.Y. Gu, W.M. Lai, V.C. Mow, A triphasic analysis of negative osmotic flows through charged hydrated soft tissues, J. Biomechanics, 30, 71-78 (1997).

11. H.S. Lew, Y.C. Fung, C.B. Lowenstein, Peristaltic carrying and mixing of chyme in the small intestine (An analysis of a mathematical model of peristalsis of the small intestine), J. Biomechanics, 4, 297-315 (1971).

12. X. Zheng; Q. Xue; R. Mittal; S. Beilamowicz, A coupled sharp-interface immersed boundary-finite-element method for flow-structure interaction with application to human phonation, ASME J Biomech. Eng., 132 (11) 111003111003-12 (2010).

13. V. Aranda, R. Cortez, L. Fauci, A model of Stokesian peristalsis and vesicle transport in a three-dimensional closed cavity, J. Biomechanics, 48, 1631-1638 (2015).

14. L. A. Taber; J. Zhang; R. Perucchio, Computational model for the transition from peristaltic to pulsatile flow in the embryonic heart tube, ASME J. Biomech Eng. 2006; 129(3):441-449. (2006).

15. A. Bertuzzi, S. Salinari, R. Mancinelli, M. Pescatori, Peristaltic transport of a solid bolus, J. Biomechanics, 16, 459-464 (1983).

16. D Tripathi, N.S. Akbar, Z.H. Khan and O. Anwar Bég, Peristaltic transport of biviscosity fluids through a curved tube: a mathematical model for intestinal flow, J. Engineering in Medicine (2016). DOI. 10.1177/095441 1916658318 (12 pages)

17. A. Tözeren, N. Özkaya, H. Tözeren, Flow of particles along a deformable tube, $J$. Biomechanics, 15, 517-527 (1982). 
18. M. Hayakawa, Y. Hosogi, H. Takiguchi, Y. Abiko, Evaluation of the electroosmotic medium pump system for preparative disk gel electrophoresis, Analytical Biochemistry, 288 (2):168-175 (2001).

19. Z. Zhu et al., A microfabricated electroosmotic pump coupled to a gas-diffusion microchip for flow injection analysis of ammonia, Micro.Acta, 182, 1063-1070 (2015).

20. H-C. Yeh, R-J. Yang, W.J. Luo, Analysis of traveling-wave electro-osmotic pumping with double-sided electrode arrays, Physical Review E, 83 (5) 056326 (2011).

21. S. Chakraborty, Augmentation of peristaltic microflows through electro-osmotic mechanisms, J. Phys. D: Appl. Phys. 395356 (2006).

22. N. T. M. El-dabe, G. M. Moatimid, M. A. Hassan, and D. R. Mostapha, Electrohydrodynamic peristaltic flow of a viscoelastic Oldroyd fluid, J. Applied Mechanics and Technical Physics, 57, 38-54 (2016).

23. J. Reece Roth, Aerodynamic flow acceleration using paraelectric and peristaltic electrohydrodynamic effects of a one atmosphere uniform glow discharge plasma, Phys. Plasmas, 10, 2117 (2003).

24. D. Tripathi, S. Bhushan and O. Anwar Bég, Transverse magnetic field driven modification in unsteady peristaltic transport with electrical double layer effects, Colloids and Surfaces A: Physicochemical and Engineering Aspects, 506, 32-39 (2016).

25. D. Si and Y. Jian, Electromagnetohydrodynamic (EMHD) micropump of Jeffrey fluids through two parallel microchannels with corrugated walls, J. Phys. D: Appl. Phys., 48, 085501 (2015).

26. D. Tripathi, S. Bhushan and O. Anwar Bég, analytical study of electro-osmosis modulated capillary peristaltic hemodynamics, J. Mechanics in Medicine and Biology 17, 1750052.1-1750052.22 (2017). DOI: http://dx.doi.org/10.1142/ S021951941750052X

27. S.S. Dukhin and B.V. Derjaguin, Electrokinetic Phenomena, John Wiley and Sons., New York (1974).

28. I. Gajda, J. Greenman, et al. Electro-osmotic-based catholyte production by microbial fuel cells for carbon capture, Water Research, 86, 108-115 (2015). 
29. A. Jamekhorshid, F. Jafarpour, G. Karimi, X. Li, Electroosmotic flow in PEM fuel cells with variable zeta potential, International Chemical Engineering Congress and Exhibition, Kish Island, 2 - 5 January (2008).

30. W.A. Gobie, C.F. Ivory, Thermal model of capillary electrophoresis and a method for counteracting thermal band broadening, J. Chromatography, 516, 191-210 (1990).

31. Y. Zhou, Y. Xie, C. Yang and Y. C. Lam, Thermal effect on microchannel electro-osmotic flow with consideration of thermodiffusion, ASME J. Heat Transfer, 137 (9) 091023-091023-10 (2015).

32. C.Y. Soong and S.H. Wang, Theoretical analysis of electrokinetic flow and heat transfer in a microchannel under asymmetric boundary conditions, J. Colloid and Interface Science, 265, 202-213 (2003).

33. A. Mukhopadhyay, S. Banerjee, C. Gupta, Fully developed hydrodynamic and thermal transport in combined pressure and electrokinetically driven flow in a microchannel with asymmetric boundary conditions, Int. J. Heat and Mass Transfer, 52, 2145-2154 (2009).

34. C-H. Chen, Heat transfer analysis of mixed electro-osmosis pressure-driven flow for power-law fluids through a microtube, ASME J. Heat Transfer, 138(8), 082001 (2016).

35. B. Cetin, D. Li, Effect of Joule heating on electrokinetic transport, Electrophoresis, 29, 994-1005 (2008).

36. A.S. Rathore, Joule heating and determination of temperature in capillary electrophoresis and capillary electrochromatography columns, J. Chromatography A,_1037, 431-43 (2004).

37. N.J. Petersen, R.P. Nikolajsen, K.B. Mogensen and J.P. Kutter, Effect of Joule heating on efficiency and performance for microchip-based and capillary-based electrophoretic separation systems: a closer look, Electrophoresis, 25, 253-69 (2004).

38. P. Vocale, M. Geri, L. Cattani, G.L. Morini, M. Spig, Electro-osmotic heat transfer in elliptical microchannels under $\mathrm{H} 1$ boundary condition, Int. J. Thermal Sciences, 72, 92-101 (2013). 
39. J.P. Escandón, O. Bautista, F. Méndez, E. Bautista, Theoretical conjugate heat transfer analysis in a parallel flat plate microchannel under electro-osmotic and pressure forces with a Phan-Thien-Tanner fluid, Int. J. Thermal Sciences, 50, 1022-1030 (2011).

40. M. Geri, M. Lorenzini, G.L. Morini, Effects of the channel geometry and of the fluid composition on the performances of DC electro-osmotic pumps, Int. J. Thermal Sciences, 55, 114-121 (2012).

41. A. Sadeghi, M. Fattahi and M. H. Saidi, An approximate analytical solution for electro-osmotic flow of power-law fluids in a planar microchannel, ASME J. Heat Transfer, 133(9), 091701 (2011).

42. Tripathi, D. (2012). A mathematical model for swallowing of food bolus through the oesophagus under the influence of heat transfer. International Journal of Thermal Sciences, 51, 91-101.

43. Li, M., \& Brasseur, J. G. (1993). Non-steady peristaltic transport in finite-length tubes. Journal of Fluid Mechanics, 248, 129-151.

\section{NOMENCLATURE}

$\begin{array}{ll}a & \text { half width of the channel }(\mathrm{m}) \\ \mathrm{c} & \text { wave velocity }(\mathrm{m} / \mathrm{s}) \\ C_{f} & \text { skin friction coefficient } \\ c_{p} & \text { specific heat at constant pressure }(\mathrm{J} / \mathrm{kg} \mathrm{K}) \\ D & \text { diffusivity of the chemical species }\left(\mathrm{m}^{2} / \mathrm{s}\right) \\ E_{x} & \text { electrokinetic body force }(\mathrm{N}) \\ g & \text { acceleration due to gravity }\left(\mathrm{m} / \mathrm{s}^{2}\right) \\ G r & \text { Grashof number } \\ \bar{h} & \text { transverse vibration of the wall }(\mathrm{m}) \\ k & \text { thermal conductivity }(\mathrm{W} / \mathrm{m} \mathrm{K}) \\ k_{B} & \text { Boltzmann constant }(\mathrm{J} / \mathrm{K}) \\ \mathrm{L} & \text { length of tube (m) } \\ \bar{n}_{+} \text {and } \bar{n}_{-} & \text {positive and negative ions, respectively } \\ n_{0} & \text { bulk concentration (number density) }\left(1 / \mathrm{m}^{3}\right) \\ N u & \text { Nusselt number } \\ \bar{p} & \text { pressure }\left(\mathrm{N} / \mathrm{m}^{2}\right)\end{array}$




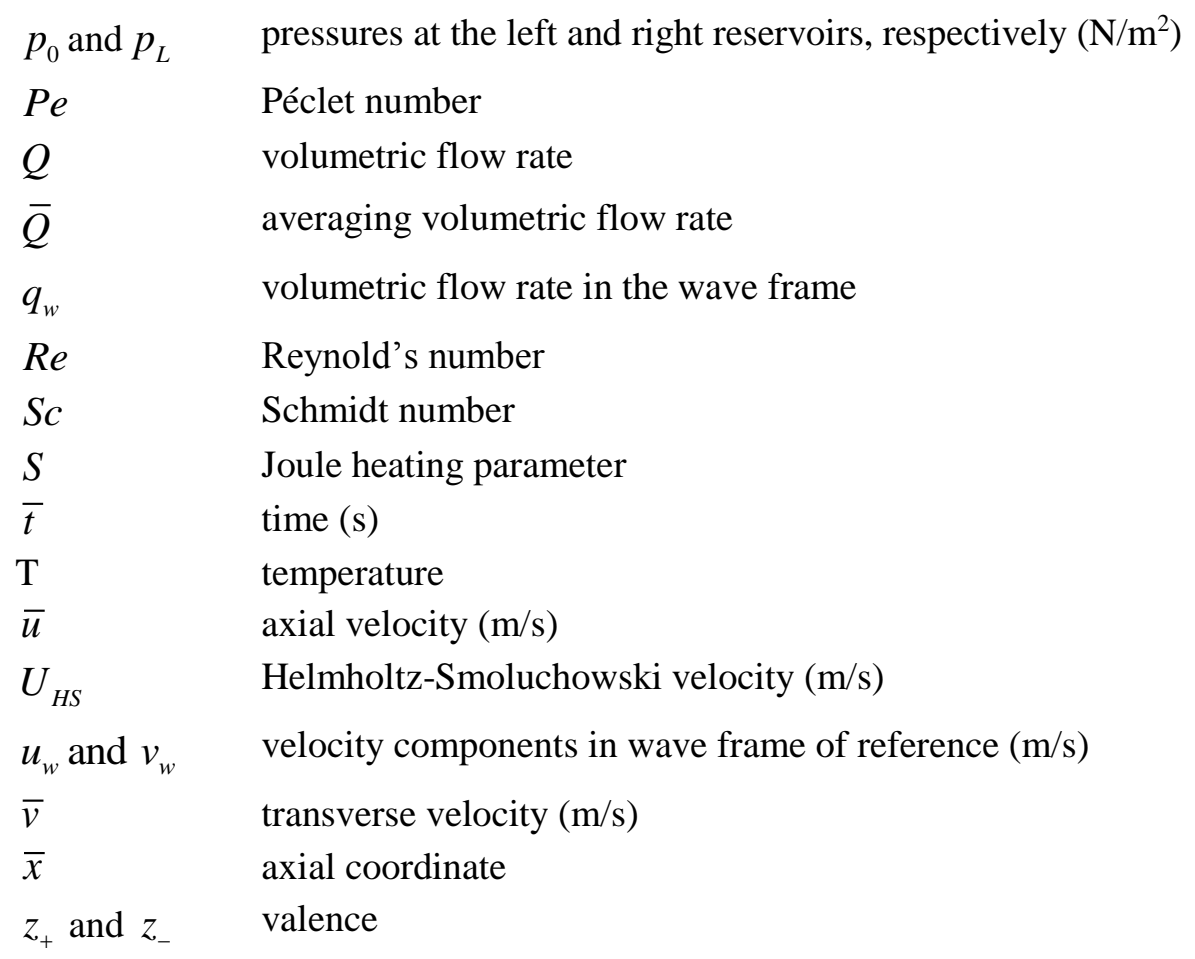

\section{Greek Symbols}

$\bar{\phi} \quad$ amplitude of the wave (m)

$\lambda \quad$ wavelength (m)

$\rho \quad$ fluid density $\left(\mathrm{kg} / \mathrm{m}^{3}\right)$

$\mu \quad$ fluid viscosity $\left(\mathrm{N} \mathrm{s} / \mathrm{m}^{2}\right)$

$\alpha \quad$ coefficient of linear thermal expansion of fluid $(1 / \mathrm{K})$

$\varphi \quad$ viscous dissipation $\left(1 / \mathrm{s}^{2}\right)$

$\Omega \quad$ heat absorption parameter $\left(\mathrm{W} / \mathrm{m}^{3}\right)$

$\sigma \quad$ electrical conductivity of the buffer fluid (S/m)

$\varepsilon \quad$ permittivity $(\mathrm{F} / \mathrm{m})$

$\bar{\rho}_{e} \quad$ electrical charge density $(\mathrm{C} / \mathrm{m})$

$\kappa \quad$ electro-osmotic parameter

$\lambda_{d} \quad$ Debye length (m)

$\theta \quad$ dimensionless temperature parameter

$\beta \quad$ dimensionless heat source/sink (absorption) parameter

$\delta \quad$ wave number

$\Delta p \quad$ pressure difference 